\title{
The common physical origin of the glass transition, macromolecular entanglement and turbulence
}

\author{
Jia-Lin Wu
}

College of Material Science and Engineering, Donghua University, Shanghai, China; ${ }^{*}$ Corresponding Author: jlwu@dhu.edu.cn

Received 27 March 2011; revised 27 April 2011; accepted 3 May 2011.

\begin{abstract}
The interface excitation (IE) on intermolecular interface is a common concept connecting the glass transition (GT), macromolecular entanglement (ME), and turbulence. IE has an additional repulsion energy and extra vacancy volume that result from the two neighboring molecules with antiparallel delocalization all in, e.g., the z-axial ground state of single-molecule instantaneous polarized dipole at GT. IEs only occur in the 8 orders of 2D IE loop-flows on local $x-y$ projection plane. Theoretical proof of the 3.4 power law of ME viscosity reveals that 1) the delocalization mode of GT and solid-liquid transition is solitary wave; wave-particle duality of solitary wave is ascribed to the equal probabilities between appearing and disappearing of IE loop-flow in inverse cascade and cascade mode; 2) macromolecular chain-length in ME motion corresponds to Reynolds number in hydrodynamics; both the ME motion and the turbulent flow obey the same scale law. IE is not the excitation of dipole energy level at GT. However, when IEs are associated with the energy levels of instantaneous polarized dipole, we predict that the coherent structure formed by multilevel 8 orders of 2D IE loop-flows is the physical origin of turbulence based on the universal random delocalization transition theory.
\end{abstract}

Keywords: Glass Transition; Mosaic Stricture; Reptation; Coherent Structure; Random Transition

\section{INTRODUCTION}

Turbulence is well-deservedly often called as "the last great unsolved problem of the classical physics." Despite enormous advances in sciences and many highly important new scientific domains emerged in the past century, the modern status of the turbulence theory is quite exceptional and differs from that of all other new sciences
[1]. On the other hand, Nobel laureate Philip Anderson wrote, "The deepest and most interesting unsolved problem in solid state is probably the nature of glass and glass transition (GT)" [2]. In physical theory, many complicated phenomena originate from the global properties (Berry's Phase) of simple quantum systems [3] and the emergent properties of the many-times repeated application of quite simple physical laws [4]. It is discovered in this paper that the physical origin of turbulence is the same as that of GT and macromolecular entanglement (ME) when solving GT and ME in polymer physics. The three famous problems all attribute to the incorporation of many physical ingredients, containing the maximal Berry's phase $\pi$ of interface excitation (IE) loop-flows and the slowest also the maximum Brownian regression-order potential in random systems. The appearance of the 8th order IE loop-flow plays the dominant role in inducing molecule-cluster localization - delocalization transition and flow speedup along one-dimensional direction in a reference $3 \mathrm{D}$ domain.

The minimum energy mode of the structure (conformational) rearrangements in supercooled liquid could be deduced from the motion mode of ME in melt liquid state. This means that we can directly use the GT theory (also proof-test the GT theory) to prove the 3.4 power law of viscosity in ME [5,6], which is a well - known experiment law found more than 60 years ago and yet failing to have theoretical proof ever since. This power law is theoretically proved in this paper. The real surprise comes when we deduce the scale law of ME motion from the power law and find that the macromolecular chain-length corresponds to the Reynolds number in hydrodynamics; both the ME motion and the turbulent flow obey the same scale law. These findings indicate that both ME and turbulence relate to the same theory and the solid-to-liquid GT is the paradigm from disorder to more disorder transition in inverse cascade - cascade mode.

Very recently, Aleiner et al. [7] show theoretically that the one-dimensional gas of short-range interacting atomic bosons in the presence of disorder can undergo a 
finite-temperature phase transition between two distinct states: fluid and insulator, and the Anderson localization - delocalization transition can be identified in the dynamics of expansion of disordered bosonic clouds released from the superimposed trapping potential. This study may also provide an existent evidence for universal random delocalization transitions presented in this paper, if we regard the energy of finite-temperature as the Brownian directional regression vibration energy along one-dimensional direction in a 3D local space and the transition between two distinct states as the inverse cascade - cascade mode of localization-delocalization transition in random systems.

This paper is organized as follows. Section 2 recapitulates the mosaic structure theory of GT, including the fixed point of self-similar two-body interaction, frustration-percolation transition; and further proves the inverse cascade - cascade mode is the fundamental mode of GT via a validity check of GT theory for the well-known abnormal exponential function in glass state. In Section 3 , we unveil the structure of ME by means of the theoretical proof for the 3.4 power law of viscosity; and show the theoretical contrast between random delocalization transition and phase transition. In Section 4, we present the theoretical predictions for the coherent structure of turbulence based on the universal geometric frustration-percolation transition theory.

\section{MOSAIC STRUCTURE}

\subsection{Inverse Cascade-Cascade Mode}

One of the GT theories is the inverse cascade-cascade mode along local one direction (e.g. notated in local $z$-axial, or on $x$-y projection plane) in a reference (thaw, excited) domain. The definition of excited domain in GT, similar to that in a ferromagnetic material, is a contiguous region in which the direction of thawing molecule-cluster spontaneous delocalization (Section 3.3) is uniform and different from that in neighboring regions. This idea comes from the insight for cooperative orientation activation energy, $\Delta E_{c o}$, on melt high-speed spinning-line [8]. When the work of the stress on over $5000 \mathrm{M} / \mathrm{min}$ spinning-line reaches $\Delta E_{c o}$, the structure of the yarn is stable and reaches full orientation, called as FOY (Full Orientation Yarn) in current polyester fiber industry. This phenomenon is called stress-induced GT. The rate of change of the stress-induced liquid-to-solid GT is $10^{7}$ times of that in general quencher from melt state to frozen glass state. A logical explanation for melt high-speed spinning is that the macromolecules can complete liquid-to-solid GT with full orientation within the millisecond of time in $z$-space. Their motion mode, within the entire range from melt transition (MT) tem- perature $T_{m}$ to GT temperature $T_{g}$, allows the direction of inverse cascade - cascade in every excited domain is in arbitrary in melt state and all apt to $z$-axial on melt spinning-line; and finally the mode is frozen in glasses as the soft matrix. At solid-to-liquid GT, increasing temperature only increases the number of IE loop-flows per unit time to augment the number of thawing domains to accelerate GT. According to the fundamental view and central assumption of de Gennes' soft matrix on mosaic structures in glasses [9], an intrinsic 8 orders of transient 2D mosaic geometric structure theory of GT has been proposed [10], Figure 1.

The structure is formed by the 8 orders of 2D IE loopflows from small to large in inverse cascade and rearrangement structure in cascade. The number of IEs on the 8th order loop is identified by means of the geometric frustration, i.e., the appearance of the 8th order directly corresponds to that of boson peak and geometric frustration in order to satisfy the claims for the theory of GT [9-12]. The number of IE of the 8th order loop equals to that of the 7 th order, and the directions of the two loops are opposite. Thus the 8 orders of 2D IE loopflows with one external degree of freedom (DoF) may be also regarded as a domain-scale interacting classical boson formed by $200 z$-component chain-particles (molecules) [10] in a $z$-axial thaw domain. Furthermore, this GT theory shows explicitly that the geometric frustration also directly corresponds to the geometric frustration-percolation transition of the 8th order loop-flow to balance the potential and the kinetic [10]. The centric 8th order $\left(V_{8}\right)$ loop one by one connects with its neighboring 47 th order $\left(V_{7}\right)$ loops in Figure 1. When the $4 V_{7}$ loops, one by one, all evolve to their $V_{8}$ loops (each $V_{8}$ loop has one external DoF); the central $V_{8}$ loop obtains 5 inner DoF and cascades to vanish (that is also the microscopic melting mode with renewed cluster energy, $k T_{m}{ }^{\circ}\left(v_{8}\right)$ ). This evolving picture holds true for all IE loops that form the flow-percolation at the GT.

In Figure 1, each (small square) chain-particle has a $z$-axial displacement energy $\Delta \varepsilon$ that comes from the Berry's phase of parallel transports of its 4 IEs surround its $z$-axis. The 8 th order loop induces each of 136 particles to hop in $z$-axial respectively at different regression times [10]. The central 5 cavity cells, in accordance with de Gennes' simple picture of "a localized 'vacancy" among clusters" [9], are also the mosaic cells denoting the 5 cooperative excited particles [10] delocalized in the correlation of sharing energy $E_{c}$ (Section 3$)$. This structure can deduce three non-integrable energies: $k T_{2}{ }^{\circ}$, $k T_{g}{ }^{\circ}, k T_{m}{ }^{\circ}$ and an icosahedral directional ordering at the GT.

The 8 orders of IE loop-flows also define the $z$-axial self-similar 8 orders of transient 2D clusters $v_{i}$ (relaxation time $\tau_{i}$ ) and 8 orders of transient 3D hard spheres $\sigma_{i}$ 


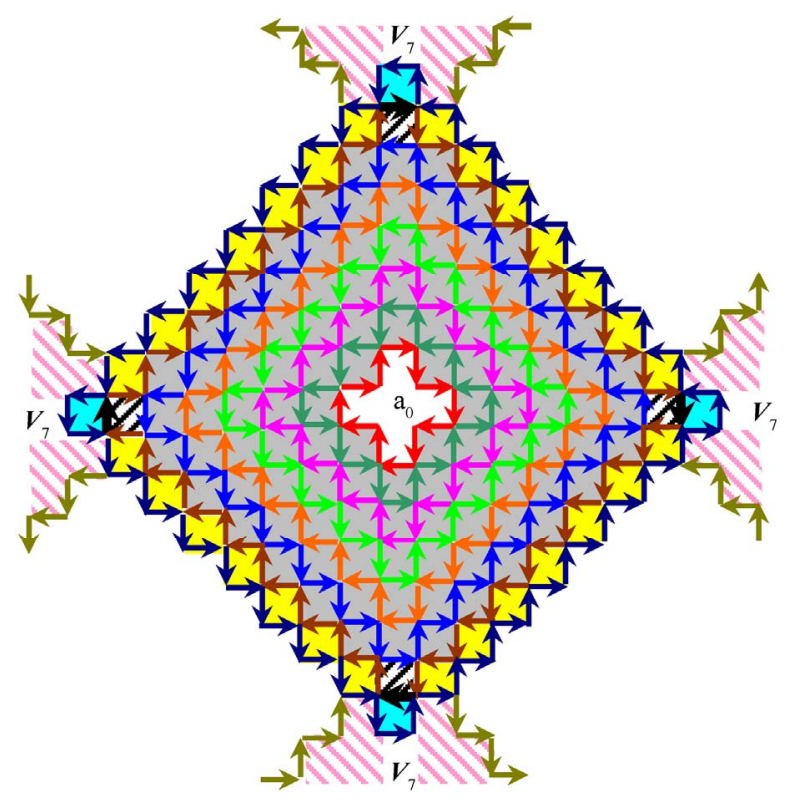

Figure 1. Sketch of the 8 orders of transient 2D mosaic structure, the maximum order potential structure in random systems.

in a thaw domain [10]. The purpose to introduce the IE concept is to find out the additional 2D transient ( $\tau_{i}$ scale) regression directional ordering potential formed by the IE loop-flows occurring respectively at the discrete local instantaneous times (Brownian regression times) $t_{i}$ in $3 \mathrm{D}$ ideal random system (the Flory disorder [13]). The additional IE energy $\Delta \varepsilon\left(\tau_{i}\right)$ with the relaxation time $\tau_{i}(i=1$, $2, \cdots, 8)$ has and only has 8 orders. The number 8 is based on the limited domain-wall vibration frequencies in the random first-order transition theories [11] of the GT. The author also independently proved that there are only 8 orders of self-similar 2D clusters $v_{i}$ and 3D hard spheres $\sigma_{i}$ at the GT [14].

The two global properties of IEs in $z$-space are found. (i) The fixed point $U_{c} *$ for 8 orders of self-similar Lennard-Jones (L-J) potentials [14], $U_{i}=f_{i} / \varepsilon_{0}\left(\sigma_{i}\right)=4\left[\left(\sigma_{i}\right.\right.$ $\left.\left(q_{i}\right)^{12}-\left(\sigma_{i} / q_{i}\right)^{6}\right], U_{c}^{*}=-15 / 16$, Figure 2. Here $\varepsilon_{0}\left(\sigma_{i}\right)$ is the potential well energy of $i$-th order cluster with directional volume $v_{i}$, or $\sigma_{i}$ and relaxation time $\tau_{i}$, so $\varepsilon_{0}\left(\sigma_{i}\right)$ also notate as $\varepsilon_{0}\left(v_{i}\right)$ or $\varepsilon_{0}\left(\tau_{i}\right), \varepsilon_{0}\left(\sigma_{i}\right) \equiv \varepsilon_{0}\left(v_{i}\right) \equiv \varepsilon_{0}\left(\tau_{i}\right)$ at GT; Generally, $\varepsilon_{0}\left(\sigma_{i}\right) \neq \varepsilon_{0}\left(\sigma_{i+1}\right)$, Figure 2 reveals that the fixed point is an universal constant for all self-similar molecule-clusters, containing the non-flexible system of $\varepsilon_{0}\left(\sigma_{i}\right) \neq \varepsilon_{0}\left(\sigma_{i+1}\right)$, with inverse cascade - cascade mode in random systems. (ii) The fixed point for reduced second Virial coefficients [15], $B_{2}\left(v_{i}\right) \equiv 3 / 8$, in Figure 3. The existences of the two fixed points have proved that the IE comes from the balance between the self-similar L-J potential fluctuations in z-axial and the (non-integrable) geometric phase induced potential fluctuation on $x-y$ projection plane. The so-called "tunneling" [16,17] results from the fact that the generating and transferring of all additional 320 IEs [10] should pass through the 8 orders of additional self-similar attractive potential center of $-17 / 16 \varepsilon_{0}\left(\tau_{i}\right),(i=1,2, \cdots, 8)[14]$, Figure 2, each of which is lower than potential well energy $\varepsilon_{0}\left(\tau_{i}\right)$ of $i$-th order cluster $v_{i} . \varepsilon_{0}\left(\tau_{i}\right)$ is also the energy of one external DoF for $i$-th order loop-flow. The theoretical proof of the well-known WLF experimental equation in polymer physics [18] validates that the energy of all the 320 IEs in the $2 \mathrm{D}$ mosaic structure is exactly the cooperative orientational activation energy $\Delta E_{c o}$ to break a solid domain [18]. This result agrees with the idea of destabilizing the crystalline state [12], and the solid-liquid coexist state evolves as the particle-flow through 8 orders of fast-slow mosaic relaxation states.

It should be pointed out that the activation energy $\Delta E_{c o}$ also correlates to the icosahedral directional ordering at GT. The icosahedral ordering [19-23] is one of the key concepts to understand the GT and the relaxation dynamics. All the 320 IEs in a local $z$-space can be also regarded as $320=20 \times 2 \times 8$. Here, $20=5$ (cooperative excited particles [10]) $\times 4$ (the number of interacting faces per chain-particle); 2 as the two-body delocalizing in $z$-space; 8 as each IE has 8 components [10], $8 \Delta \varepsilon=\varepsilon_{0}$, the energy of one external DoF that can be experimentally detected at the GT.

The experimental value of IE energy for flexible system is $\Delta \varepsilon_{0}\left(\tau_{i}\right)=\Delta \varepsilon \approx 5.6 \times 10^{-4} \mathrm{eV}$ from WLF equation [18]; and $\Delta \varepsilon \approx 5.5 \times 10^{-4} \mathrm{eV}$ from $\Delta E_{c o}$ on melt spinning-line $[8,10]$. So $\varepsilon_{0}\left(\tau_{i}\right)=8 \Delta \varepsilon \approx 4.4 \times 10^{-3} \mathrm{eV}$. This value is consistent with the experimental results $(4 \sim 12$ $\times 10^{-3} \mathrm{eV}$ ) for Boson peak measured by high-resolution inelastic neutron scattering [12]. For non-flexible general

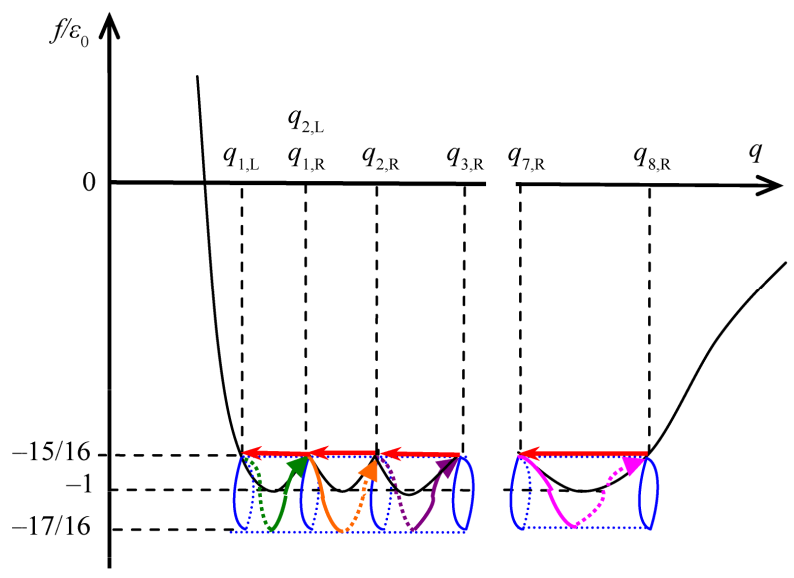

Figure 2. Two-body delocalization along 8 orders of geodesic, the fixed point of $-15 / 16 \varepsilon_{0}\left(\sigma_{i}\right)$ is the universal constant for all self-similar molecule-clusters $\sigma_{i}$ with inverse cascade-cascade mode in random systems. 
system, each of 320 IEs may have different IE energy. Thus, each material has its own two inherent values of independent of temperature in its own random system: the geometric frustration-percolation (also the localization-delocalization) transition energy, $E_{c}=k T_{g}^{\circ}\left(v_{i}\right)$, and the renewed cluster energy, $k T_{m}{ }^{\circ}\left(v_{i}\right) . k T_{g}{ }^{\circ}\left(v_{i}\right)$ is also the non-integrable directional regression vibration (DRV) kinetic energy of $i$-th order cluster $v_{i}, k T_{g}{ }^{\circ}\left(v_{8}\right)=k T_{g}$ in inverse cascade; and $k T_{m}{ }^{\circ}\left(v_{i}\right)=k T_{g}{ }^{\circ}\left(v_{i}\right)+4 \varepsilon_{0}\left(v_{i}\right), k T_{m}{ }^{\circ}\left(v_{8}\right)$ $=k T_{m}$, of the 8 orders of clusters in cascade [10].

\subsection{Origin of Interface Excitation in Statistical Physics}

This theory differs from current mode-coupling theories [11] of GT. A phenomenological concept of IE is introduced by the ripplon and the universal Lindemann distance increment [10] $d_{L}$ in order to obtain the global properties of IEs in inverse cascade - cascade. The spirit in mode-coupling scheme deals with the coupling of fast-slow relaxation modes and two density modes in structure rearrangements at GT. In our mode-coupling scheme, we first focus on finding out the three direction non-integrable energies $k T_{2}^{\circ}, k T_{g}^{\circ}$ and $k T_{m}^{\circ}$ existing in the coordinates invalidation from $i$-th order clusters to $(i$ $+1)$-th order at GT, in spite of the time complications in all anharmonic frequencies. This means that the crucial $2 \mathrm{D} 2 \pi$ closed loop with the maximum non-integrable Berry's Phase plays a major role in anharmonic frequency. The mode-coupling trick is that the relaxation time complications have been beforehand reduced and replaced by the slow Brownian directional regressions. Then we deduce from global to local to find out the microscopic origin of IE (Section 2.3). The mosaic structure theory of GT reaches a new level by revealing the microscopic physical origin of IE.

The fluctuation stability condition in (ii) is that the chemical potentials are always zero in all subsystems [15]. There is a profound theoretical contrast between chemical potential and 'temperature'. The chemical potential is defined as Fermi energy level on the occasion when the random kinetic energy of temperature in system is zero in solid physics; whereas the non-integrable random DRV energy, $k T_{g}^{\circ}$, in a thaw domain, is defined as the delocalization energy, $E_{c}$, on the occasion when the chemical potentials in all subsystems are always zero at the solid-to-liquid GT. The key here is that the $k T_{g}{ }^{\circ}$ is only comprised by the slowest (z-space) DRV energy, which is exactly the lowest-frequency $\left(\tau_{i}\right.$ scale $)$ mode that has been explored at the GT. The $x$-axial and $y$-axial ( $\tau_{i}$ scale) DRV energy of all $z$-component molecules (chain-particles) encircled by IEs should be zero in order to minimize the total dominant GT energy.

In Figure 2, there are 8 sharp-angled points, $U_{c} *=$
$U\left(q_{i, R}\right)=U\left(q_{i+1, L}\right)$ [14], forming the delocalization paths of two $z$-axial (i.e. $q$-axial in the figure) $\sigma_{1}$ clusters along 8 orders of geodesic with IE energy $\Delta \varepsilon\left(\tau_{i}\right)=1 / 8 \varepsilon_{0}\left(\tau_{i}\right)$. $i$-th order geodesic is the shortest line of $2 \pi$ cycle between $q_{i, R}$ and $q_{i+1, R}$ on $i$-th order of cylindric potential surface (on $x-y$ projection plane, taking $\varepsilon_{0}=-1$ as $z$-coordinate axis). The red arrows denote the paths of structure rearrangements in cascade: from $q_{8, R} \rightarrow q_{7, R}$ $\rightarrow \cdots \rightarrow q_{1, R} \rightarrow q_{0, R}$

\subsection{Absence of Attraction in 2D Lattices}

Thus, two $\sigma_{1}$ clusters are in the delocalization state, absent of attraction in vibration, along 8 orders of geodesic. The two molecule-clusters in the absence of Van der Waals attraction are all through in a repulsion state on the $x-y$ projection plane during generating their one IE. Van der Waals interaction includes the contribution of instantaneous induced dipole - induced dipole.

Generally, instantaneous polarized dipole electron charges randomly distribute on interface 1-2 forming electron cloud (blue zone) in Figure 3(a).

At GT, an interesting and unexplored corner in Van der Waals interaction theories is that the synchronal instantaneous polarized electron charge coupling pair (two small blue dots) may parallel transport on an interface 1-2 between chain-particles $a_{0}$ and $b_{0}$, or the interface 2-3 between $a_{0}$ and $c_{0}$ to form IE in Figure 3(b), or 3(c). Since the site-phase difference on $x-y$ plane between the two z-component molecules is $\pi$ [10], the state of the all polarized electron charges in each instantaneous dipole, in the two $z$-component molecules in Figure 3(b-1) or 3(c-1), must be in the same state and in the $z$-axial minimum energy state (ground state) of single-molecule instantaneous dipole. In Figure 3, (b-2) and (c-2) is respectively the projection of (b-1) and (c-1) on $x-y$ plane. Thus, the additional IE energy is the repulsive energy between the two instantaneous synchrony $z$-axial ground state polarized electron charges that parallel transport, from one end to other end on an interface in Figure 3(b-2), simply denoted by an arrowhead $(1 \rightarrow 2)$ in $\mathbf{3 ( d )}$ on local $x-y$ projection plane. Note that the two IE states of (b-1) and (c-1) in Figure 3 occur at different local instantaneous times. The directions of the next two parallel transports of instantaneous polarized electron charge of the reference $a_{0}$ are denoted as two red-broken line arrowheads in Figure 3(c-1). IE loop-flow $1 \rightarrow 2 \rightarrow$ $3 \rightarrow 4 \rightarrow 1$ in Figure 3(d) offers a non-integrable potential $\Delta \varepsilon$ to induce ion $a_{0}$ (the center of mass of $a_{0}$ particle) $z$-direction a displacement $\Delta_{z}$. Black-broken line arrow denotes the delocalizing direction of each ion in Figure 3. Figure 3 explains the origin of 5-particle cooperative excited field in [10]. The molecules in the absence of the $x$-, $y$-axial $\left(\tau_{i}\right.$ scale) vibrations are in the high density 


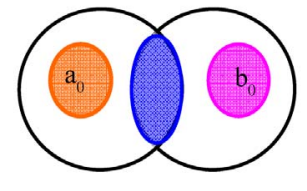

(a)

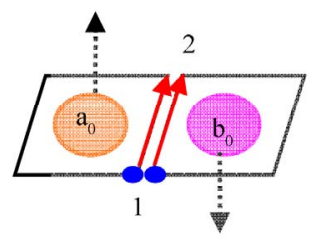

(b-2)

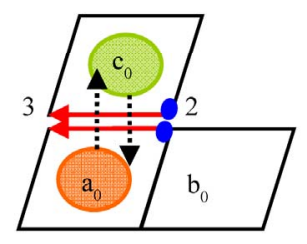

1

$(\mathrm{c}-2)$

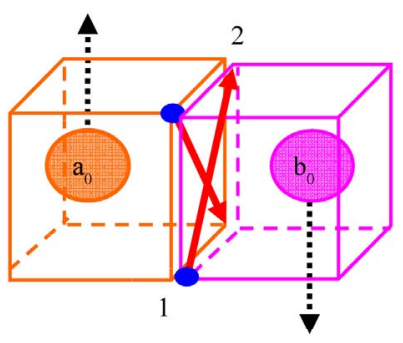

(b-1)

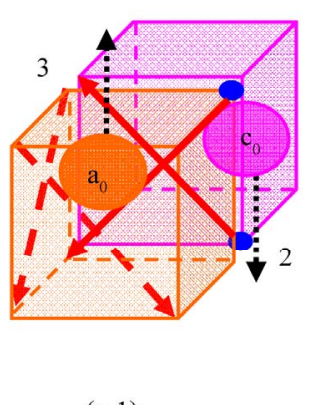

(c-1)

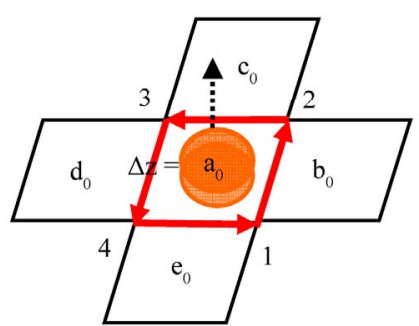

(d)
Figure 3. The microscopic physical origin of IE. (a) A legitimate state; (b), (c) An absent of attraction state in 2D lattice; (d) delocalizing instantaneous dipole state.

state, which agrees with de Gennes' picture of the compact primary clusters [9].

\subsection{Random Delocalization Transition}

A region of space that can be identified by a single mean field solution is called a mosaic cell [24]. The IE loop-flow can expediently affirm interfaces of mosaic cell. Due to the effect of geometric frustration - percolation transition, the number of IEs on the 8th order loop is corrected as 60 [10]. Based on the corrected value we can also validate the theory of IE. By single mean field solution, we directly obtain the percolation (also the delocalization) energy $E_{c}=20 / 3 \varepsilon_{0}$ [10]. Since the energy in inverse cascade - cascade is not dissipated, we also directly get the 8 orders of non-integrable potentials $E_{c}\left(\tau_{i}\right)$ $=20 / 3 \varepsilon_{0}\left(\tau_{i}\right)$ to induce 8 orders of clusters $v_{i}$ inverse cascade along local one direction. The balance between $E_{c}\left(\tau_{i}\right)$ and $k T_{g}{ }^{\circ}\left(\tau_{i}\right)$ is realized by the fixed point in statis- tical physics, the fixed point of reduced second Virial coefficients $B_{2}\left(T^{*}\right)$ for self-similar clusters $v_{i}$ in different size. That is, $B_{2}\left(T^{*}\right)=B_{2}\left(k T_{g}{ }^{\circ} / \varepsilon_{0}\right) \equiv 3 / 8$ at the GT, in which $k T_{g}^{\circ}\left(\tau_{i}\right) \approx 20 / 3 \varepsilon_{0}\left(\tau_{i}\right)$ in Figure 4, independent of temperature and time, and $k T_{g}^{\circ}\left(v_{8}\right) \equiv k T_{g}^{\circ}\left(\tau_{8}\right)=k T_{g}$.

In Figure 4, $T^{*}=k T / \varepsilon_{0}\left(\sigma_{i}\right)$, is the reduced temperature in [25], also the reduced non-integrable DRV energy in our discussion. Graphical method gives the only set of approximate solution, $B_{2}\left(T_{g}{ }^{*}\right) \approx 3 / 8, T_{g}{ }^{*} \approx 20 / 3$, satisfies the self-similar Eq.5 for the curve of $B_{2}\left(T^{*}\right)_{L-J}$. The numerical solution refers to the result in [25].

In inverse cascade at the GT, in the percolation evolution fields from cluster $v_{i-1}$ to $v_{i+1}$, we may furthoer rewrite the reduced Virial expansion as the form of Eq.1 in [15].

$$
P V\left(v_{i+1}\right) / k T\left(v_{i}\right)=B_{2}\left(v_{i}\right)+B_{3}\left(v_{i-1}\right)
$$

Where, potential takes cluster volume $v_{i+1}$ as variable which denotes that the induced potential is fast motion whereas the molecule-clusters moving is slow motion. The phase difference is invariable as $\pi$ between kinetic and potential. The result of two-body interaction always gets evolvement volume to $v_{i+1}$ from $v_{i}$. Two-body interaction is slower than three-body. In other words, three -body is always firstly compacted in order to minimize the totally IE energy. The two-body interaction is in fact the interaction of two three-bodies in fluctuation at the GT [15].

In statistical mechanics, the abnormal thermal capacity occurs in self-similar system. From enthalpy $H=E+$ $P V$, the definition of Joule-Thomson coefficient [25] $\mu_{j}$ is

$$
\mu_{J}=(\partial T / \partial P)_{H, N}=C_{P}^{-1}\left[T(\partial V / \partial T)_{P, N}-V\right]
$$

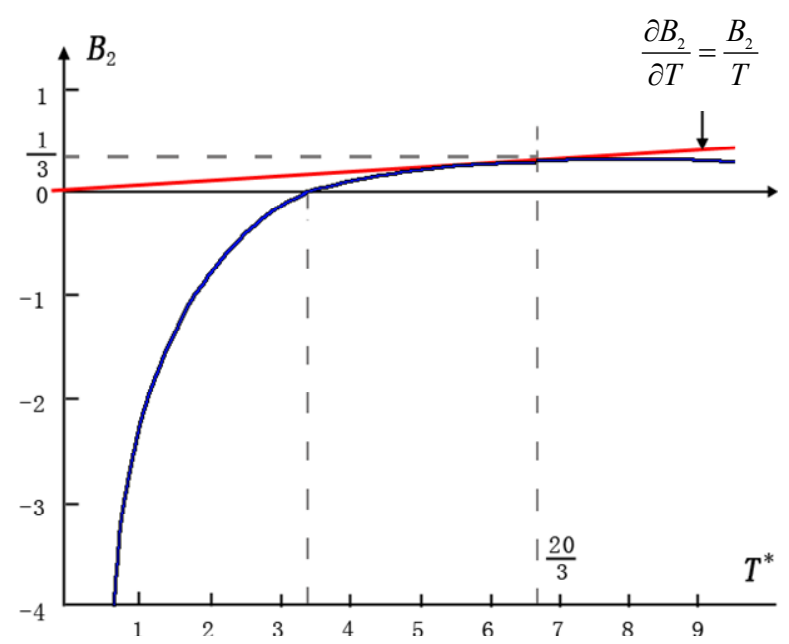

Figure 4. The fixed point $\left(B_{2}{ }^{*}, T^{*}\right) \approx(1 / 3,20 / 3)$ of self-similar reduced second Virial coefficients validates for all directional molecule-clusters. $T^{*}$ here is the reduced non-integrable DRV energy in random systems. 
Assume the state of $\mu_{J} \equiv 0$ corresponds to GT. When $\mu_{J} \equiv 0$

$$
(\partial V / \partial T)_{P, N} \equiv V / T
$$

Rewrite as Eq.4 for $v_{i}$ cluster

$$
\left(\partial V\left(v_{i}\right) / k \partial T\left(v_{i}\right)\right)_{P, N} \equiv V\left(v_{i}\right) / k T\left(v_{i}\right)
$$

Here $k T$ in (2) - (4) should be also regarded as the non-integrable random DRV energy of $i$-th order cluster $v_{i}$ in $z$-space. The energy $k T$ also is a function of the self-similar cluster volume $v_{i}$, when outside pressure (stress) remains constant. As long as the condition $\mu_{J} \equiv 0$ in (2) is satisfied and (4) also holds, $C_{P}$ in the case of (4) may also show an abnormal change. We see the abnormal thermal capacity may occur in the following two cases. One is in the polymer GT, $C_{P}$ occurs at the temperature $T_{g}$. In this case, the $k T_{g}\left(=E_{c}\right)$ is the percolation energy. The other is in the low-temperature GT in local domain region, the abnormal thermal capacity occurs in the manner of the energy of Boson Perk.

The hard-sphere (square well potential with IE energy) model at the GT can be also deduced from the 8 orders of self-similar L-J potentials along the clusters inverse cascade direction [14]. It can be strictly proved that the reduced third Virial coefficient for hard-sphere system is constant, $B_{3} \equiv 5 / 8,[25,26]$ independent of temperature and cluster volume. Thus, in $z$-space of the clusters inverse cascade, we can also get $B_{3}\left(v_{i-1}\right) \equiv 5 / 8$. Therefore, in the percolation evolving field from cluster $v_{i-1}$ to $v_{i}$, from the interaction of two three-bodies, we have

$$
P V\left(v_{i}\right) / k T\left(v_{i}\right)=B_{2}\left(v_{i}\right)
$$

in $z$-space fluctuation. In $z$-space, from (1),

$$
\frac{\partial B_{2}\left(v_{i}\right)}{\partial V\left(v_{i}\right)}=\frac{P}{k T\left(v_{i}\right)}=\frac{B_{2}\left(v_{i}\right)}{V\left(v_{i}\right)}
$$

From (4), we obtain an important self-similar Eq.5 at the GT in [15].

$$
\frac{\partial B_{2}\left(v_{i}\right)}{k \partial T\left(v_{i}\right)}=\frac{B_{2}\left(v_{i}\right)}{k T\left(v_{i}\right)}
$$

It Figure 4, if we regard the energy of $T_{g} *$ as the $z$-space reduced DRV kinetic energy of 8 orders of self-similar clusters, the point $\left(B_{2}, k T_{g}^{\circ}\right) \approx\left(3 / 8,20 / 3 \varepsilon_{0}\right)$ is exactly the universal geometric frustration - percolation transition energy for any cluster volume $v$ in random delocalization transitions. Moreover, the $B_{2} \equiv 3 / 8$ at the GT is directly proved by scaling theoretical approach [15].

$$
\frac{P V\left(v_{i+1}\right)}{k T\left(v_{i}\right)}=B_{2}\left(v_{i}\right)+B_{3}\left(v_{i-1}\right) \equiv 3 / 8+5 / 8 \equiv 1
$$

where $i=1,2, \cdots, 7$, and when $i=8$, the induced poten- tial $E_{c}$ directly equals to $k T_{g}$ in the geometric frustration - percolation transition at GT. Eq.6 holds true on all subsystems (flow-percolation fields), which means that the kinetic energy always keeps balances with the potential energy, in the manner of the maximum Berry's phase of $\pi$, in the mode of 8 orders of coupling 2body-3body fluctuation clusters at GT.

\subsection{Order-Induced Molecule Delocalization}

The concept of connecting Anderson transition and GT is the percolation limit model. In the theory of Anderson disorder-induced (electron) localization [13], the competition between kinetic energy and potential energy influences on the electron states can reside in the ratio $W / B, W$, the magnitude of the random potential, and $B$, the (crystal) bandwidth in the absence of disorder. A classical method of demonstrating consequences of disorder is the percolation model [13]. At the GT, the percolation transition energy is $E_{c}\left(\tau_{8}\right)=E_{c} . \Delta E_{c o}$ is the orientation activation energy with 320 IEs on the 8-order 2D mosaic structure in local $z$-space [10]. The ratio of $E_{c}$ $/ \Delta E_{c o} \equiv \phi_{c}\left(E_{c}\right) \equiv 1 / 6$, its physical meaning is that $\phi_{c}\left(E_{c}\right)$ specifies the occupied fraction of $z$-space 320 IE states that allow flow of energy $E_{c}$ to occupy. The reason of the cooperative molecules obtaining the probability of delocalization is that they share energy $E_{c}$. The ratio is consistent with the result (the classical analog for electron delocalization) of Zallen [13], who suggests $\phi_{c}\left(E_{c}\right) \approx$ $0.16 \approx 1 / 6$, for the percolation limit on a continuum in $3 \mathrm{D}$ space. Here, the occupied fraction of all IE states allowed to flow of energy $E_{c}$, takes the place of the occupied fraction of space allowed to molecules of energy $E_{c}$. This means that there is the inherent theoretical contrast between molecule delocalization and electron localization on the concept of percolation limit.

That is, from the viewpoint of percolation, there are two classical percolation limits. One is the limit of the maximum disorder for the potential in random corresponds to the Anderson transition, that is the Zallen's viewpoint [13]; whereas, the other is the limit of the maximum order for the potential in random to the GT. At the GT, all molecules encircled by 4 IEs on $x-y$ projection plane are in the minimum excited energy state (states), i.e., at the $z$-axial ground state of single - molecule dipole in the absence of $x$-, $y$-axial ( $\tau_{i}$ scale) vibrations. The DRV induced potential energy in 5-particle cooperative excited field [10] can be also regarded as the overall order potential limit (in contrast to the maximum disorder potential limit in Anderson random) in the random Brownian motion. That is the reason we call the GT as the order-induced molecule (molecule-cluster) delocalization transition in random systems.

In other words, the delocalization energy $E_{c}$ at the GT 
comes from the maximum order energy in random and the minimum additional ground-state repulsive energy. Thus, the percolation limit mode (the crossover from the site- or bond- percolation in solid to the flow-percolation in liquid) of the GT can probably explain why the 8 orders of 2D mosaic geometric structure (Figure 1) associated with percolation transition so simple. That is, this nano-scale soft mosaic structure reflects the nature of random delocalization transition in random systems.

\subsection{Percolation Limit Picture at GT}

The theory of IE loop-flow directly and definitely shows $E_{\text {mig }}=k T_{2}=17 / 3 \varepsilon_{0} . E_{\text {mig }}$ is defined as the average energy of cooperative migration along one direction in $a$ percolation field [10], independent of temperature. During $\left[t_{i}, t_{i}+\Delta t_{i}\right]$ in a reference domain, the entire transient $\tau_{i}$ scale displacement energy in $\pm z$-axial is $(136 / 8) \varepsilon_{0}=$ $17 \varepsilon_{0}$, Figure 1, and the $\tau_{i}$ scale vibration energy in $x$-, $y$-axial is zero; thus, the average DRV kinetic energy is also $17 / 3 \varepsilon_{0}=k T_{2}$. On the other hand, during $\left(t_{i}+\Delta t_{i}, t_{i+1}\right)$, the average random energy in the domain is as $k T_{2}$. However, the average DRV kinetic energy would occur in other domains in the system during $\left(t_{i}+\Delta t_{i}, t_{i+1}\right)$ in the reference domain at the GT. This is the dual-role of $k T_{2}$ in $k T_{g}$, reflecting the balance between random DRV energy and random thermal motion energy at the condition of $k T_{g}{ }^{\circ}$. If take $E_{m i g}=k T_{2}$ as the average DRV kinetic energy in dual-role, $k T_{2}$ is in fact the deferred action DRV kinetic energy until the appearance of the 8th order 2D loop (the appearance of $k T_{g}$ ). In this case, $k T_{2}$ will be of the DRV kinetic energy of the 8th order cluster in the absent of energy $\varepsilon_{0}\left(\tau_{8}\right)$ in an excited domain in Figure 1. Vice versa, when the temperature is $T_{2}$, the reference domain will also be of the probability of regression order potential of $k T_{2}$ if the domain can obtain the energy $\varepsilon_{0}\left(\tau_{8}\right)$. Thus, the physical meaning of $k T_{2}$ is that in a $3 \mathrm{D}$ solid-domain, taking any reference direction, as long as superadded the energy $\varepsilon_{0}\left(\tau_{8}\right)$ on the domain, the spontaneous molecules delocalization would be occur in the reference direction.

Its singularity different from phase transition is the non-ergodicity that the adding energy may be either a few energies only on several domains, or a large energies one by one on neighboring domains to form a flow-percolation field. In any case, if the adding energy $\varepsilon_{0}$ denoted by temperature, it always satisfies $k T_{2}+\varepsilon_{0}=k T_{g}$. In other words, here $\varepsilon_{0}=\varepsilon_{0}\left(\tau_{8}\right)$ is a reduced energy to reduce the magnitude of its action region or its action time in the system. The noticeable contrast between phase transition and random transition in physical theory (Table 1) is as follow. At Curie temperature point, $k T_{c}$, in magnetism, so as decrease a little of random energy $\Delta(k T)$ in whole system, the spontaneous magnetiza- tion will occur along one direction in whole system. Whereas, at $k T_{2}$ of the GT, so as increase a little of $d i$ rectional ordering energy $\varepsilon_{0}$ in a local region, the molecules spontaneous delocalization will occur along the direction in the local region. It is in this sense $T_{2}$ corresponds to Curie temperature in magnetism. The numerical value of $17 / 3 \varepsilon_{0}$ is also validated by the WLF experimental equation [18], 17/3 $\varepsilon_{0}+\varepsilon_{0}=k T_{g}=20 / 3 \varepsilon_{0}=E_{c}$. There are two critical delocalization energies $E_{c}$ and $k T_{2}$. $E_{c}$ is the critical percolation transition energy, corresponding to the spontaneous delocalized solitary wave (Section 3) in percolation field (the "ocean" in percolation theory) at GT; and $k T_{2}$ is the critical energy of particle-clusters delocalization at low-temperature GT in local domain scale (the "lake" in percolation theory) in percolation limit model. This percolation limit picture with separate 'lakes' and 'ocean' is distinctly different from the general percolation model. Note: the delocalizing step-size in glassy is far less than the amplitude of thermal vibration, Section 3.3, thus the glass state in random delocalizing is still in stable state.

\subsection{Soft Matrix and Emergent Property}

The de Gennes' central assumption of the soft matrix [9] is also validated. The soft matrix is exactly $i$-th $(i \leq 7)$ order 2D loop-flows frozen in glass state. From which the low-temperature GT would occur through a longer Brownian regression time to inverse cascade until the 8 th order and to cascade at low-temperature. The lower the frozen, the longer is the required time of inverse cascade. Therefore, the abnormal exponential function [27] in glass state can be proved directly.

Generally, the physical quantity $\xi(t)$ in a system will return according to the physical law of Eq.7 when the system deviates from its equilibrium state.

$$
\xi(t) \sim \exp \left[-\left(\frac{t}{\tau}\right)\right]
$$

However, if the glassy system is driven (or normally fluctuates) out of equilibrium, it returns according to the formula [28]

$$
\exp \left[-\left(\frac{t}{\tau}\right)^{\beta}\right]
$$

Where $t$ is the (system) time and $\tau$ and $\beta$ are parameters. Unfortunately this is not a mathematical expression that is frequently encountered in physics. So little idea exists of what the underlying mechanisms are [28].

Since the inverse cascade-cascade motions only occur in some discrete "lakes" in glass state when $T<T_{g}$. Eq.7 still holds true in these "lakes" regions as long as the $t$ in (7) is the local domain time. One of the key concepts is 
that the equilibrium state of glass state is the equilibrium state between the random thermo motion energy $k T$ and the slowest DRV energy. DRV energy always dominates the number of the excited domains at temperature $T$. Assume the glass state we observed is the nonequilibrium state that comes from the equilibrium state at the temperature $T_{1}$ suddenly drops to the temperature $T_{2}$ at the time $t=0$, and $T_{2}<T_{1}<T_{g}$. During the relaxation time of $t$, the entire DRV relaxation energy is $\varepsilon=k\left(T_{1}-\right.$ $\left.T_{2}\right)=k \Delta T$. From the famous Kolmogorov law in cascade $[1,29]$

$$
\frac{l_{i}^{2}}{t_{i}^{3}}=\dot{\varepsilon}_{i}=k \Delta T / t=\text { constant } .
$$

Where $l_{i}$ is the length scale of $i$-th order of loop (cluster) and $\dot{\varepsilon}_{i}$ is the cascade energy mobility; $t_{i}$ is the local domain time and $t$ is the relaxation time in system. From (8)

$$
t_{i}=\left(\frac{l_{i}^{2} t}{k \Delta T}\right)^{\frac{1}{3}}
$$

Substituting $t_{i}$ in (9) for $t$ of local domain time in the right term on (7)

$$
\xi(t) \sim \exp \left(-\frac{l_{i}^{2} t}{k \Delta T \tau_{l o c}}\right)^{\frac{1}{3}}=\exp \left[-\left(\frac{t}{\tau_{s y s}}\right)^{\beta}\right]
$$

The physical meaning of $\beta$ is very definitude, $\beta=1 / 3$ denotes the cascade motion in ideal glass state in the case of the small fluctuation value of $k \Delta T$. The reason of the deviation from $1 / 3$ for $\beta$ may be that (a) the influence of the competition between the change ratio of temperature $\mathrm{d} T / \mathrm{d} t$ and the 8 orders of relaxation times on IEs; (b) the larger value of $k \Delta T$ conduces $\beta$ augment because of the inverse cascade always lower than cascade. Similarly, if the nonequilibrium glass state is arose by outside stress (or electromagnetism and other factors) work $\Delta W$, the relaxation of $\Delta W$ can only realize through the inverse cascade - cascade in the excited domains as same as that in the proof of WLF equation [18]. In this case, we only need replace $k \Delta T$ by $\Delta W$ in (9). This means that the abnormal mathematical expression (10) in glass state is also only the emergent property of domains in system, as same as the abnormal expression of WLF equation in the GT (the many-times repeated applications of Clapeyron equation governing first order phase transition on the subsystems will educe WLF equation) [18]. The general physical relaxation law (7) still holds true in domain scale in glass state. The theoretical proof also confirms that inverse cascade-cascade mode is the fundamental mode for glass state.

\section{ENTANGLEMENT STRUCTURE}

\subsection{Theoretical Proof of the 3.4 Power Law}

The viscosity in ME melt is $\eta \sim N^{3.4}$ for all linear entangled polymers. Since $N$ is a large number, the experimental 3.4 power law of viscosity has sensitivity for any modified theory to de Gennes reptation model. In concordance with the experimental 3.4 power law of viscosity, within the experimental error range of viscosity, we first roughly estimate the theoretical error tolerance by adopting a fine theory described ME viscosity. The critical entanglement chain length $N_{c}=200[11,30]$. The experimental value of chain length $N$ is generally less than $10^{3}$ (seeing the Fig. 9.5 in [5]). If taking the range of $N$ as $200 \sim 1000, N^{3.4} / N^{3.3} \approx 1.7(N=200) \sim 2$ $(N=1000)$. This indicates the error between theoretical value and experimental result of viscosity will be about $70 \% \sim 100 \%$ if the theoretical value as 3.3 ; and $N^{3.4} /$ $N^{3.35} \approx 1.3 \sim 1.4$. This means that a fine theory should be able to give the theoretical exponential value range of viscosity as $3.4 \pm 0.05$ for flexible polymers. At the same time, we expect this theory must also be able to predict the exponential of viscosity that is in line with the experimental results for non-flexible polymers. This is also a fine way to check up the GT theory. The existing various modified theories [5] cannot match for the 3.4 power law. This exponent is significantly large than the prediction of 3 by de Gennes reptation model [5,30] based on the assumption that the chain of length $N$ is a "free chain". The reason of the deviation is the required number of DoF of one-step-walk along z-axial for each chain particle depends on $N_{z}$ the $z$-component of chain $N$. The key of the theoretical proof is to find out the number of DoF, $N^{*}$, for chain-length $N$. Only through substitution of the reference chain of length $N$ in single-chain reptation model with an equivalent particle-chain of length $N^{*}$, can the random diffuse motion of the reference chain $N$ be entirely free in tube model. Thus, in the 'equivalent multichain' de Gennes reptation model, we have

$$
\eta \sim\left(N^{*}\right)^{3}
$$

The one-step-walk along $+z$-axial of a reference particle $a_{0}$ on chain $N_{z}$ results from the induced action of the 8 orders of 2D loop-flows on the $\mathrm{x}-\mathrm{y}$ projection plane in $a_{0}$ (local excited) field. Only when the 8th order appears and acts on the particle $a_{0}$, should one "particle-cavity" in $+z$-direction appear in $a_{0}$ field and bring $a_{0}$ to move one step along $+z$-axial. Statistically, when $N_{z}>N_{c}=200$ ( $N_{c}$ is also the number of the chain-particles of structure rearrangements $[10,11,30,31]$ in the 8 orders of $2 \mathrm{D}$ mosaic structure), the moving of the 200 cooperative particles along $z$-space on $x-y$ projection plane in $a_{0}$ field 
should share the energy $E_{c}$ with $a_{0}$. Due to the sharing energy effect, even if $a_{0}$ has obtained a cavity with one-step-walk, the motion of $a_{0}$ should still be correlated with the 200 particles (molecules) in shared energy $E_{c}$. In other words, the cooperative particles still "drag" the one-step-walk of $a_{0}$.

The external DoF of the 8th order 2D loop-flow encircled $a_{0}$ is 1 , taking $\varepsilon_{0}\left(\tau_{8}\right)$ as the energy unit of DoF. In order to eliminate the correlation of share energy to make $a_{0}$ move freely in $z$-axial, let $E_{c} / \varepsilon_{0}\left(\tau_{8}\right)=L_{g}$, where $L_{g}$ is the equivalent number of particles. $E_{c}$ thus is as the "loop-flow" energy with an equivalent chain of length $L_{g}$ circling $a_{0}$. On the one hand, from the viewpoint of particle $a_{0}$ on chain $N_{z}$, statistically, the $N_{c}$ chain-particles on $N_{z}$ also share the energy $E_{c}$. Thus, the probability that $a_{0}$ is possessed of one unit DoF on the chain $N_{z}$ is $1 / N$. On the other hand, from the viewpoint of the $L_{g}$ equivalent particles, statistically, each equivalent particle is located at its own long-chain with length $N_{z}$, and the probability that each equivalent particle is possessed of one unit DoF on its own long-chain is also $1 / N$.

When particle $a_{0}$ on chain $N_{z}$ is substituted by an equivalent chain of length $L_{g}$, the probability of the event that it obtains one unit DoF and freely moves one step along $z$-axial, denoted as $p_{+}\left(a_{0}\right) \cdot p_{+}\left(a_{0}\right)$ is namely the probability of the event that all the $L_{g}$ equivalent particles simultaneity move one step along z-direction in the co-Brownian motion by $200 N_{z}$ chain-particles in $a_{0}$ field. So, $p_{+}\left(a_{0}\right)$ is given as

$$
p_{+}\left(a_{0}\right)=(1 / N)^{L_{g}}
$$

Statistically, the probability of each particle freely moving one step in z-space should be all equal to $p_{+}\left(a_{0}\right)$. To maintain the balance of motion between inverse cascade and cascade in $\mathrm{z}$-space, the probability that one reference 8th 2D loop-flow disappears in cascade in co-Brownian motion by $200 N_{z}$ chain-particles is denoted as $p_{-}\left(a_{0}\right)$ and obtained as follows.

Let $a_{0}$ have $n_{z}$ DoFs with $\varepsilon_{0}\left(\tau_{8}\right)$ as the unit in cascade motion. $n_{z}$ is actually the number of DoF to make conformational rearrangement of the chain $N_{z}$ in z-space, which results from the contribution of the reference 8th order 2D loop-flow transferred from the particle $a_{0}$ field to the other particle field on the chain. Similar to (12), we have

$$
p_{-}\left(a_{0}\right)=\left(n_{z}\right)^{L_{m}}
$$

where $L_{m}=k T_{m} / \varepsilon_{0}\left(\tau_{8}\right), L_{m}$ is the number of 'equivalent particles' to eliminate the reference 8 th order $2 \mathrm{D}$ loop-flow of $a_{0}$ in cascade.

In the complex inverse cascade-cascade motion, the equilibrium condition is

$$
p_{+}\left(a_{0}\right)=p_{-}\left(a_{0}\right)=p
$$

so,

$$
n_{z}=\left(\frac{1}{N}\right)^{\frac{T_{g}}{T_{m}}}
$$

The number of DoF of $N_{z}$ chain-particles on chain of length $N_{z}$ to make conformational rearrangement in $z$-space is $N_{z}^{*}, N_{z}^{*}=n_{z} N_{z}$,

$$
N_{z}^{*} \propto N^{\left(1-\frac{T_{g}}{T_{m}}\right)}
$$

In (16), $T_{g}^{\circ}$ has been approximately replaced by $T_{g}$. $N_{z}^{*} \varepsilon_{0}\left(\tau_{8}\right) \equiv H$, here $H$ is enthalpy in (2), the constant energy for rearrange conformation (that also the energy of $z$-space solitary wave in Section 3.3) in random system. So, during the reptation of long-chain $N$ in the 3D space, the number of DoF $N_{x}^{*}$ in the $x$-space and $N_{y}^{*}$ in the $y$-space have $N_{z}^{*}=N_{y}^{*}=N_{x}^{*}$, or

$$
N^{*} \propto N^{3\left(1-\frac{T_{g}}{T_{m}}\right)}
$$

From (11) and (17), we obtain the general power law expression of viscosity in $\mathrm{ME}$

$$
\eta \propto N^{9\left(1-\frac{T_{g}}{T_{m}}\right)}
$$

For flexible chain polymers, $k T_{m}=k T_{g}+4 \varepsilon_{0}\left(\tau_{8}\right), k T_{g}=$ $20 / 3 \varepsilon_{0}\left(\tau_{8}\right)[10]$. We have

$$
N^{*}=N^{\frac{9}{8}}
$$

And $\eta \propto N^{\frac{27}{8}}=N^{3.375} \approx N^{3.4}$

This theoretical result conforms well with the experimental data. For non-flexible chain polymer, Eq.18 can be verified. For example, for the polypropylene (PP), the $T_{g}(\mathrm{PP})$ is $-10^{\circ} \mathrm{C}=263 \mathrm{k}[32]$; the $T_{m}(\mathrm{PP})$ is $176^{\circ} \mathrm{C}=$ 449k [33]; Eq.18 given $\eta_{p p}$ (theory) $\sim N^{3.73}$, conforms well with the experimental data $\eta_{p p}($ experiment $) \sim N^{3.72}$ [34].

The theoretical proof further confirms that inverse cascade-cascade mode is the fundamental mode in the solid-to-liquid transition whether in macromolecular or in small molecular.

\subsection{Degrees of Freedom of Chain-Length $\mathbf{N}$}

Since cascade is also the mode in turbulent flow, we discuss the total number of DoF, $N_{L}$, in the cube with edge length of $L\left(L \geq N^{*}\right)$ in ME melts. The unit for the length of $L$ here is the loop scale, $l_{s}$, of the 8 th order 2D loop-flow. Due to the properties of flow-percolation [10], an 8th order 2D loop-flow may be in arbitrary direction.

In other words, in 3D space, we can consider any one 
"random-walk chain" with "chain-length" $N_{L}$ and each "chain-particle" with one unit length $l_{s}$. If the "end-to-end distance" of the "random-walk chain" is always equal to $L, L$ is then the root-mean-square of end-to-end "chain-length" $N_{L}$, that is, $N_{L}=L^{2}$, in Figure 5.

When $L \geq N^{*}, N_{L} \sim\left(N^{*}\right)^{2}$, from (19), we have

$$
N_{L} \sim N^{9 / 4}
$$

Comparing to the famous Kolmogorov relationship [29] between the number of DoF $N_{R}$ and the Reynolds numbers $R_{e}$ in (a uniform grid) turbulence

$$
N_{R} \sim R_{e}^{9 / 4}
$$

It can be seen that the two terms in two different subjects: turbulence and ME both describe the same complex motion phenomena about inverse cascade - cascade. Both of them obey the same scaling law, which indicates there is a universal theory behind them. Reynolds number is actually the ratio of the inertia force to the drag force, and the macromolecular chain-length reflects the ratio of the number of the particles "inertially" one by one sharing the delocalization energy $E_{c}$ to the one particle entangled with them in motion.

\subsection{Solitary Wave}

Each physical quantity in (12) - (18) is the inherent invariable in random system for every material, independent of temperature and time. Eqs.15 and 16 reveal a $z$-space solitary wave moving $N_{\mathrm{z}}$ steps along a random long-chain $N_{z}$ from one end to other end. A distinct characteristic of the solitary wave is that the "particle" energy of each one-step-walk is "quantized": $n_{z} \varepsilon_{0}\left(\tau_{i}\right)=n_{z} \varepsilon_{0}$ for flexible system. While $n_{z} \varepsilon_{0}\left(\tau_{i}\right)$ generally have 8 orders of components, 8 orders of potential well energies for general non-flexible system. The step-size is only the $n_{z}$ particles of size. The number of step of its traveling wave is $N_{z}=N$. The reptation in 3D space of a ME chain is assembled in time by the three solitary waves on the chain in $x$-, $y$ - and $z$-space, which in fact attributes to the co-Brownian regression motion by $200 \mathrm{~N}$ chain-particles respectively in $x$-, $y$ - and $z$-space.

Accordingly, the ME structure about viscosity is in fact the 8 orders of transient 2D mosaic structures on $x-y$ projection plane and the delocalized solitary wave in $z$-space. $n_{z}$ is a small numerical value, e.g. if taking $N=$ $N_{c}=200, n_{z} \approx 0.036$. However, Eq.15 reveals that $n_{z}$ connects with the length-boundaries $N_{z}$ of solitary wave in its each one-step-walk. The velocity of traveling wave will be determined by other factors including, e.g., the generating ratio of IEs by temperature, time or outside stress work, and the magnitudes of material relaxation times in IEs. This special characteristic of solitary wave shows that it differs from the mode of conventional quan-

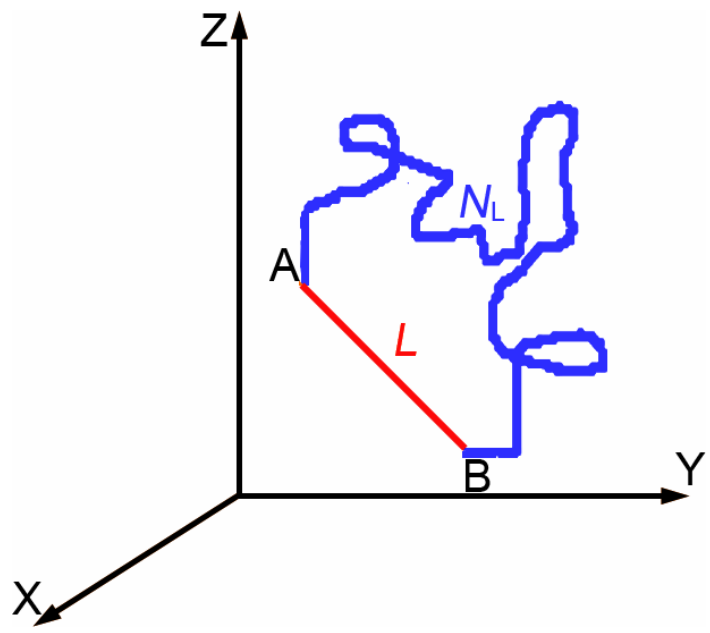

Figure 5. The relationship between "chain-length" $N_{L}$ and number of degrees of freedom.

tum-mechanical wave; the latter is always obtained from the combination of both the wave equation and the boundary conditions.

\subsection{Spontaneous Symmetry Breaking at GT}

The paradigm for the phase transition theory is the ferromagnetic transition. de Gennes [30] emphasized that polymer solutions should compare with magnets. However, difficulty occurs in the order parameter for a polymer solution [30]. de Gennes proposed that the order parameter is the magnetization of a spin system with a number of spin components $n=0$ [30]. Edwards' more concrete statement is that the order parameter $\Psi$ is similar to a quantum mechanical creation (or destruction) operator [30]. The proof for 3.4 power law predicts a definitude result for the remaining problem that the order parameter is the spontaneous delocalized solitary wave $\Psi$ of a classical spin system (8 orders of 2D IE loops) with a number of additional instantaneous spin components $n=0$, whether in macromolecules or in small molecules, within the entire range from solid to liquid transition. The physical meaning of $n=0$ is here that the additional "spin" of a reference chain-particle only comes from the contributions of its 4 neighbouring particle fields, instead of its own inner-rotation change. In addition, $n=0$ also corresponding to self-avoiding random walk [30] of 8th order 2D loop-flows.

Contrary to phase transition, we have unveiled the step by step way from disorder to more disorder in Table 1. In which we have implied that the disordered degree (measurement) depends on the maximum 2D IE loop-size and the number of the loops in a random system. The conjugate variables of solid-to-liquid transition in Table 1 are deduced from the results of de Gennes in [30]. 
Table 1. The contrast between delocalization transition and phase transition in 3D space.

\begin{tabular}{|c|c|c|}
\hline Critical point & Ferromagnetic phase transition & Solid-to-liquid delocalization transition \\
\hline Number of spin components $n$ & $n=3$ & $n=0$ \\
\hline Cell & Magnetic moment $S$ & displacement energy $\Delta \varepsilon$ circled by 4 IEs \\
\hline Spontaneity & Magnetization $M$ & Delocalizing solitary wave $\psi$ \\
\hline "Curie" point & $T_{c}$ & $k T_{2}$ : critical delocalization energy \\
\hline Reduced energy increment $\varepsilon$ & $\begin{array}{c}\varepsilon=k\left(T-T_{c}\right) / k T_{c}, \varepsilon>0, M=0 \text { in system } \\
\varepsilon \leq 0, M=\langle S\rangle \neq 0 \text { in system }\end{array}$ & $\begin{array}{c}\varepsilon=k\left(T-T_{2}\right) / \varepsilon_{0}, \varepsilon_{0}: \text { potential well energy } \\
\varepsilon=1, \mathrm{GT}: \psi \neq 0 \text { in subsystems } \\
\varepsilon<1, \text { Low-temperate GT: } \psi \approx 0 \text { in domain } \\
\varepsilon=5 \text {, MT: Reptation in subsystems } \\
1<\varepsilon<5 \text {, Viscoelasticity (Intermittency) }\end{array}$ \\
\hline Symmetry breaking & $M$ chooses one-space, breaking the isotropic $M$ & $\begin{array}{l}\text { GT: Solitary wave chooses one space, breaking the } \\
\text { isotropic thermal motion } \\
\text { MT: Three solitary waves in } x, y, z \text {-space restore the } \\
\text { isotropic thermal motion }\end{array}$ \\
\hline Self-similar cluster & cluster size $\xi_{v} \rightarrow \infty$ & Finite 8th order cluster \\
\hline Correlation length $\xi$ & $\xi \rightarrow \infty$ & $\begin{array}{c}\xi=N_{\mathrm{z}}, \text { macromolecular chain-length } \\
\xi=N_{c}, \text { for small molecule systems }\end{array}$ \\
\hline Order parameter & $M$ & Solitary wave $\Psi$ \\
\hline Replica symmetry & Spin wave & 8 orders of 2D IE loop-flows \\
\hline $\begin{array}{l}\text { Conjugate variables Extensive } \\
\text { quantity Intensive quantity }\end{array}$ & $\begin{array}{l}M \\
H\end{array}$ & $\begin{array}{c}\left.N_{\mathrm{z}} \text { (or } N_{c}\right) \text {, step number of solitary wave } \\
n_{\mathrm{z}} \varepsilon_{0}, \text { one-step-energy of solitary wave }\end{array}$ \\
\hline Generalized rigidity & Magnetic hysteresis & 8th order 2D IE loop \\
\hline Defect & Domain boundary & Extra vacancy volume in each IE on 8th order loop \\
\hline
\end{tabular}

\section{COHERENT STRUCTURE}

\subsection{The Way to Turbulence}

Thus, we have ascribed the GT problem to the systematics of the special parallel transport state of bonding on intermolecular interface. This is precisely in line with the insight in [28] that crucial to the endeavor of GT theory is a deeper understanding of the systematics of bonding in condensed matter within a framework going considerable beyond the current GT picture. All that we have done is to replace the GT problem by the bonding problem of parallel transport of instantaneous polarized electron charges containing all atoms (atom-clusters) in a chain-particle at the GT. However, we have progressed and can now discuss turbulence.

The IE and the 8 orders of 2D mosaic structures at GT may be called as the ground state IE and the first level of 8 orders of 2D mosaic structures. For small molecule system, due to the energy transfer of rearrangement conformation between two $z$-axial connecting molecules, $N_{c} z$-component molecules respectively in absence of $x$-, $y$-axial vibrations can also be connected one by one to form a $z$-space random "long-chain with chain-section as a molecule" walking $N_{c}$ steps in the correlation of sharing energy $E_{c}$.

Solid-to-liquid transition does not invoke the energy level of molecule (molecule-cluster) instantaneous induced dipole. However, for each energy level of molecule (molecule-cluster) instantaneous polarized dipole, e.g., for the first energy level of instantaneous polarized electron charges of molecule (of all atoms and molecules in a molecule-cluster), two neighboring molecules with antiparallel delocalization may be all in the $z$-axial first energy level state of single-molecule instantaneous polarized electron charges on an intermolecular interface. Such IE is named as the first-energy-level IE. A new 8 orders of 2D mosaic structure can be formed, which is called the second level of 8 orders of 2D coherent (mosaic) structure.

For small molecule liquid, only about $4 / 15\left(8 k T_{m} /\right.$ $\left.\Delta E_{c o}=4 / 15\right)$ interfaces have been excited. As disorder increases, more ground state IE will be excited to accelerate liquid flow, which corresponds to the ordinary flow development.

As disorder keeps increasing and flow continues speedup, each ground state IE on the 8th order 2D loop can update to be the first-energy-level IE with new 8 orders of relaxation times, new additional IE energy and vacancy volume. All other IEs on $x-y$ projection plane are still in the ground state of IE. All these $z$-component molecules in the absence of $x$-, $y$-axial vibrations can form a $z$-space random "long-chain" with "chain-section" as 136 molecules encircled by 60 first-energy-level IEs. 
60 first-energy-level IEs can form a 0-th "cluster-particle" in $z$-space in the second level of 8 orders of $2 \mathrm{D}$ loop-flow, denoted as $v_{0}^{(1)}\left(a_{0}\right)$, the figure in the superscript parentheses denotes the first-energy-level IE of single-molecule polarized electron charges. The 0 -th "cluster-particle" is self-similar to the $z$-component molecule $a_{0}$ [named as $\left.v_{0}\left(a_{0}\right)\right]$ in Figure 1. The size of the 0 -th "cluster-particle" is that of the 8th order cluster $v_{8}\left(a_{0}\right)$ in the first level of mosaic structures. The 0 -th "cluster-particle" has also 4 "interfaces" with the side length of 15 IEs (one thick-black inverted arrow plus the 14 sky-blue arrows in Figure 1) of the first energy level. Thus, the second level of 8 orders of 2D mosaic structures is self-similar to the first level. To drive the 136 molecules cooperatively move one step along $+z$-space only needs the energy $n_{z}{ }^{(1)} \varepsilon_{0}{ }^{(1)}$. The mode is the solitary wave with the first-energy-level IE in $z$-space.

\subsection{Multilevel of Random Localization - Delocalization Transitions}

Inverse cascade is the accumulation of the directional Brownian regression potential energy in loop-flows, and cascade is the transfer of energy from potential to kinetic to drive liquid cluster flow. The larger loop is formed in inverse cascade, the bigger liquid cluster is driven in cascade, and the higher efficiency the system has to excite liquid flowing, and the faster the velocity of flow is.

In the same way, when the flow keeps on speedup, the third, the forth...the $l$-th level of 8 orders of 2D coherent structures can be constructed one by one, in order to augment the loop scale and the loop-potential so that the maximum 2D loop-potential reaches the value of the potential forced by environment to the liquid flow. At the moment, the $l$-th level geometric frustration - percolation transition (seeing the universal pictures of Figures 1 and 2) appears and reaches potential balances with kinetic, and the cascade phenomenon of the maximum loop-flow begins. This is the turbulent flow. The origin of the intermittency turbulence [35] is that the high non-ergodic state of flowing arises suddenly to make the reduced energy increment, $\varepsilon^{(l)}$, of each level with a value slightly more than 1 , similar to the viscoelasticity in Table 1. The heteroclinic orbits [36] in turbulence may be the flow-lattice edges of the $l$-th level 2D coherent structures.

The complicated turbulence can come down to the $l$-th level solitary wave in $z$-space and the $l-1$ reptations (snake-walks) of $l-1$ various levels (self-similar subsystems) simultaneously occurring in 3D space.

At critical phase transition, the self-similar cluster size is $\xi_{v} \rightarrow \infty$, while it is only $N_{c}$ in GT, Table $\mathbf{1}$, which is also the localization-delocalization scale of the first level in universal random delocalization transitions. In other words, on the way from disorder to more disorder, the cluster size of $\xi_{v} \rightarrow \infty$ in critical phase transition will be here divided up by $l$ levels, $N_{c}^{(l)}$, corresponding to the cooperative localization - delocalization scale of $l$-th level, i.e. it is also divided into $l$ levels of geometric frustration-percolation transitions, similar to Figures 1-5, in random localization - delocalization transitions on the way from disorder to more disorder. This is the way to turbulence.

\section{CONCLUSIONS}

A theoretical perspective on the mosaic structure theory of GT has been proposed. The theoretical approach of GT connects closely the origin of turbulence. A new and central concept is the instantaneous parallel transport repulsive state absent of attraction in 2D lattices in current Van der Waals interaction theories. The solid to liquid GT is the paradigm of the inverse cascade - cascade mode and the first level of the way to disorder and turbulence. Nine physical ingredients, random, selfsimilar, two-body interaction, fluctuation, frustration, percolation, delocalization, Berry's phase (parallel transport) and Brownian regression potential, have been incorporated on IE loop-flows at the GT and the universal random delocalization transition.

The theoretical proofs for the abnormal exponential function in glass state and the 3.4 power law of viscosity confirm that the inverse cascade-cascade mode is the fundamental mode whether in solid to liquid transition or in macromolecular motion. Due to the effect of incorporation of physical ingredients, the different terms in different subjects have been also incorporated. The inverse cascade-cascade mode with mosaic structure reflects the confluence of both the kinetic dimensions and the thermodynamics at the GT. It can be also denoted by both the delocalization solitary wave (that is also the melt mode, the conformational rearrangement mode, structure rearrangement mode) along a local one-dimensional direction and the 8 orders of $2 \mathrm{D}$ IE loop-flows (its scale or size is also a domain scale, cage scale, local scale, correlation scale of two-body interaction, random localization-delocalization transition scale, maximum step-scale of random walk, geometric frustration scale and critical percolation scale in random systems) on the local projection plane.

Three different structure terms, the mosaic structure in GT, the entanglement structure in ME and the coherent structure in turbulence, are all in fact the transient 2D geometric structure formed by IE loop-flows in their own random system. The most important physical quantity correlating GT, ME and turbulence is the directional ordering induced potential of a maximum 2D IE loopflow in random system. Once the loop-flow is formed, 
the GT appears, the critical ME phenomenon arises, and the cascade in turbulence begins.

\section{ACKNOWLEDGEMENTS}

The author is grateful to all colleagues he had the pleasure to collaborate and interact, especially when he found the fundamental physics origin for the orientation activation energy obtained experimentally on melt high- speed spinning-line in 1986. In particular, the author would like to thank, in random order, Yuan Tseh Lee and Sheng Hsien Lin of Academia Sinica (Taiwan), Yun Huang of Beijing University, Da- -Cheng Wu of Sichuan University for useful discussions. Support from the Academia Sinica (Taiwan), and the State Key Lab of Chemical Fibers and Polymer Materials, Donghua University (Shanghai) is gratefully acknowledged.

\section{REFERENCES}

[1] Lesieur, M., Yaglom, A. and David, F. (2002) The trends of turbulence. Springer, Berlin.

[2] Anderson, P.W. (1995) Through the Glass Lightly. Scence, 267, 1615-1616. doi:10.1126/science.267.5204.1615-e

[3] Berry, M.V. (1998) Foreword to "Global properties of simple quantum systems - Berry's phase and others". http://www.phy.bris.ac.uk.

[4] Kadanoff, L.P. (1991) Complex Structures from Simple Systems. Physics Today, 44, 9. doi:10.1063/1.2810017

[5] Rubinstein, M. and Colby, R.H. (2003) Polymer physics. New York Publisher, Oxford.

[6] Doi, M. and Edwards S.F. (1988) The theory of polymer dynamics. New York Publisher, Oxford.

[7] Aleiner, I.L., Altshuler, B.L. and Shlyapnikov, G.V. (2010) A finite-temperature phase transition for disordered weakly interacting bosons in one dimension. Nature Physics, 6, 900-904. doi:10.1038/nphys 1758

[8] Wu, J.-L., Guan, D. and Quian, B. (1986) The characteristic behaviour of the stretch-orientation zone during high-speed PET spinning. International Polymer Processing, 1, 25-31.

[9] Gennes, P.G. (2002) A simple picture for structural glasses. Comptes Rendus Physique, 3, 1263-1268. doi:10.1016/S1631-0705(02)01387-7

[10] Wu, J.-L. (2009) Unified model theory of the glass transition: (Part 1) intrinsic 8 orders of 2D mosaic geometric structure. Journal of Materials Science and Engineering, 3, 69-83.

[11] Lubchenko, V. and Wolynes, P.G. (2003) Origin of the boson peak and thermal conductivity plateau in low temperature glasses. Proceedings of the National Academy of Sciences, 100, 1515-1518. doi:10.1073/pnas.252786999

[12] Greaves, G.N., Meneau, F., Majérus, O., Jones, D.G. and Taylor, J. (2005) Identifying vibrations that destabilize crystals and characterize the glassy state. Science, $\mathbf{3 0 8}$, 1299-1302. doi:10.1126/science. 1109411

[13] Zallen, R. (1983) The physics of amorphous solids. Wiley, New York.

[14] Wu, J.-L. (2009) Unified model theory of the glass transition: (Part 2) fixed point of self-similar Lennard-Jones potentials in the glass transition. Journal of Materials Science and Engineering, 3, 58-64.

[15] Wu, J.-L. (2009) Unified model theory of the glass transition: (Part 3) fixed point of second virial coefficients in the glass transition. Journal of Materials Science and Engineering, 3, 31-39.

[16] Laird, B.B. and Bembenek, S.D. (1996) Localization and the Glass Transition, Journal of Physics: Condensed Matter, 8, Article ID: 9569. doi:10.1088/0953-8984/8/47/064

[17] Barkai, E., Naumov, E.A., Vainer, Yu.G., Bauer, M. and Kador, L.L. (2003) Statistics for random single-molecule line shapes in a glass. Physical Review Letters, 91, 1-4. doi:10.1103/PhysRevLett.91.075502

[18] Wu, J.-L. (2009) Unified model theory of the glass transition: (Part 4) Theoretical proof of the standard WLF equation in the glass transition. Journal of Materials Science and Engineering, 3, 76-82.

[19] Mechler, S., Macht, M.-P., Schumacher, G., Zizak, I. and Wanderka, N. (2010) Frustration of the stable Zr-Ti-Ni quasicrystal as the basis of glass formation. Physical Review B, 81, 1-4. doi:10.1103/PhysRevB.81.180101

[20] Coslovich, D. and Pastore, G. (2007) Understanding fragility in supercooled Lennard-Jones mixtures: I. Locally preferred structures. Journal of Chemical Physics, 127, 1-13. doi:10.1063/1.2773716

[21] Shin, H.K. (2010) Vibrational relaxation of NO- $(v=1)$ in icosahedral (Ar) 12NO-clusters. Journal of Chemical Physics, 132, Article ID: 104302. doi:10.1063/1.3339385

[22] Tanaka, H. (2003) Roles of local icosahedral chemical ordering in glass and quasicrystal formation in metallic glass formers. Journal Physics: Condensed Matter, 15, 491-498. doi:10.1088/0953-8984/15/31/102

[23] Jain, T.S. and de Pablo, J.J. (2005) Local structure and motions in a supercooled polymer. Journal of Chemical Physics, 122, Article ID: 174515. doi:10.1063/1.1888505

[24] Lubchenko, V. and Wolynes, P.G. (2003) Barrier softening near the onset of nonactivated transport in supercooled liquids: Implications for establishing detailed connection between thermodynamic and kinetic anomalies in supercooled liquids. Journal of Chemical Physics, 119, Article ID: 9088. doi:10.1063/1.1614180

[25] Reichl, L.E. (1980) A modern course in statistical physics. University of Texas Press, Dallas.

[26] Pathria, R.K. (1977) Statistical mechanics. Pergamon Press, Oxford.

[27] Williams, G. and Watts, D.C. (1970) Non-symmetrical dielectric relaxation behavior arising from a simple empirical decay function. Transactions of the Faraday Society, 66, 80-85. doi:10.1039/tf9706600080

[28] Physics Survey Committal (1986) Physics through the 1990s, condensed-matter physics. National Academy, Washington.

[29] Frisch, U. (1995) Turbulence. Cambridge University, Cambridge.

[30] de Gennes, P.G. (1985) Scaling concepts in polymer physics. Cornell University, New York.

[31] Lubchenko, V. and Wolynes, P.G. (2001) Intrinsic quantum excitations of low temperature glasses. Physical Review Letters, 87, 1-4. doi:10.1103/PhysRevLett.87.195901

[32] Kent, J.A. (2007) Riegel's handbook of industrial chem- 
istry and biotechnology. Vol. 1, 11th Edition, Springer, New York.

[33] Frank, H.P. (1986) Polypropylene. Gordon and Breach Science, New York.

[34] Zhao, D., Fan, Q., Quian, R. and Xiu, D. (1981) Relationship between the zero shear rate viscosities of PP melt and its viscosity average molecular weights. Bulle- tin of Macromolecula, 5, 385-388.

[35] Wright, W.B., Budakian, R., Pine, D.J. and Putterman, S.J. (1997) Imaging of intermittency in ripple-wave turbulence. Science, 278, 1609-1216. doi:10.1126/science.278.5343.1609

[36] Vassilicos, J.C. (2001) Intermittency in turbulence flows. Cambridge University, Cambridge, 44-63. 